\title{
Perturbations of Schwarzschild black holes in laboratories
}

\author{
E. Abdalla. * R.A. Konoplyat and A. Zhidenkd \\ Instituto de Física, Universidade de São Paulo \\ C.P. 66318, 05315-970, São Paulo-SP, Brazil
}

\begin{abstract}
It is well-known that the perturbations of Schwarzschild black holes are governed by a wave equation with some effective potential. We consider perturbations of a gas in a tube called the de Laval nozzle, which is narrow in the middle and has a sonic point in the throat. By equating the wave equation in a de Laval nozzle of an arbitrary form with the wave equation of spin-s perturbations of Schwarzschild black holes, we find the exact expression for the form of the de Laval nozzle, for which acoustic perturbations of the gas flow corresponds to the general form of perturbations of Schwarzschild black holes. This allows observation, in a laboratory, of the acoustic waves, which are analogue of damping quasinormal oscillations of Schwarzschild black holes. The found exact acoustic analog allows to observe also some other phenomena governed by the wave equation, such as the wave scattering and tunneling.

PACS numbers: 04.30.Nk,04.50.+h
\end{abstract}

\section{INTRODUCTION}

One of the most essential characteristics of black holes are the resonant frequencies of the response to external perturbations, called quasinormal frequencies [1]. Being an analog of normal modes for open systems, they do not depend on the way in which the system is perturbed, but only on the parameters of a system. Quasinormal modes are expected to be observed in the near future with the help of a new generation of gravitational antennas. It is well-known that non-rotating astrophysical black hole can be described by the Schwarzschild solution, implying the importance of the quasinormal modes of this background [2].

In addition to the still elusive possibility to observe quasinormal modes (QNMs) of black holes with the help of a new generation of gravitational antennas, there is a window for observation of the acoustic analogue of a black hole in laboratories. This is the well-known Unruh analogue of black holes [3], which are the apparent horizons appearing in a fluid with a space-dependent velocity, in the presence of sonic points. The supersonic waves cannot propagate back beyond the sonic point, mimicking thereby, the effect of the horizon at sonic points in a membrane paradigm. The Unruh discovery stimulated active investigation of quasinormal modes of different analogue black holes [6]. First of all, the quasinormal spectrum of the analogue black holes given by the metrics:

$d s^{2}=-\left(1-C / r^{2}\right) d t^{2}+\left(1-C / r^{2}\right)^{-1} d r^{2}-2 B d \phi d t+r^{2} d \phi^{2}$

and

$$
d s^{2}=-\left(1-r_{0} / r^{4}\right) d t^{2}+\left(1-r_{0} / r^{4}\right)^{-1} d r^{2}+r^{2} d \Omega^{2}
$$

\footnotetext{
*Electronic address: eabdalla@fma.if.usp.br

${ }^{\dagger}$ Electronic address: konoplya@fma.if.usp.br

${ }^{\ddagger}$ Electronic address: zhidenko@fma.if.usp.br
}

were considered [ [6]. These are the two models for rotating analogue black holes in "draining bathtub" and for a canonical analogue non-rotating black holes. Recently, interesting acoustic analogues of brane-world black holes were suggested [7, [8].

We can see from the above formulas that these metrics, even being very useful as analogues with apparent horizons, do not represent a true solutions of the Einstein or other gravity dynamical equations. If one had a complete analogy with some known solution of Einstein equations, say, the Schwarzschild solution, he could see in the acoustic experiments not only qualitative, but also, up to an experimental accuracy, exact numerical coincidence with a prototype characteristics. Namely, for quasinormal modes, which are governed by the form of the wave equation, this numerical correspondence would mean that the effective potential of the perturbations of some hydrodynamic system coincides with an effective potential of a black hole. Fortunately, recent consideration of the perturbations of a gas in de Laval nozzle [4] gives us such an opportunity of finding a system that is realted to the same effective potential as a Schwarzschild black hole.

The canonical de Laval nozzle is a convergent-divergent tube, narrow in the middle. It allows to accelerate the gas until the sonic speed in its throat, reaching supersonic speeds after passing the throat. The perturbations of the gas in de Laval nozzle can be considered as onedimensional if the section of the nozzle does not change too quickly. Here we show that the corresponding effective potential of perturbations in a canonical de Laval nozzle can be made to be equal to the potential for perturbations of Schwarzschild black holes, if choosing some specific form of the nozzle. In addition, we suggest another, approximate, way to get quasinormal modes of Schwarzschild black holes in de Laval nozzle. For this, one needs to mimic the form of the effective potential for Schwarzschild metric with the help of a de Laval nozzle of a simple form suggested in [4].

The paper is organized as follows: Sec 【 gives all ba- 
sic equations of the one-dimensional motion in de Laval nozzle we shall use. Sec III is devoted to reproducing the exact expression for the form of the nozzle which corresponds to the potential of the Schwarzschild black holes. In the discussions, we sketch the open questions and possible generalizations of the suggested technique.

\section{CALCULATION OF THE NOZZLE CROSS SECTION $A$ IN TERMS OF $g$}

We assume that a gas in the nozzle can be described by equations of motion for perfect fluid and that the flow is quasi-one-dimensional:

$$
\begin{gathered}
\partial_{t}(\rho A)+\partial_{x}(\rho v A)=0, \\
\partial_{t}(\rho v A)+\partial_{x}\left[\left(\rho v^{2}+p\right) A\right]=0, \\
\partial_{t}(\epsilon A)+\partial_{x}[(\epsilon+p) v A]=0,
\end{gathered}
$$

Here $\rho$ is the density, $v$ is the fluid velocity, $p$ is the pressure, $A$ is the cross section of the nozzle, and

$$
\epsilon=\frac{1}{2} \rho v^{2}+\frac{p}{\gamma-1}
$$

is the energy density. The heat capacity ratio is $\gamma=$ $1+2 / n=7 / 5=1.4$ for di-atomic molecules of air $(n=5)$. We shall assume that the flow has no entropy discontinuity, then the fluid is isentropic

$$
p \propto \rho^{\gamma}
$$

Instead of Eq. (2), we can use Euler's equation

$$
\rho\left(\partial_{t}+v \partial_{x}\right) v=-\partial_{x} p
$$

For isentropic fluid Eq. (6) is reduced to the Bernoulli's equation

$$
\partial_{t} \Phi+\frac{1}{2}\left(\partial_{x} \Phi\right)^{2}+h(\rho)=0
$$

where $h(\rho) \equiv \int \rho^{-1} d p$ is the specific enthalpy and $\Phi=$ $\int v d x$ is the velocity potential.

According to [4], the perturbation equations in such a nozzle can be reduced to:

$$
\begin{gathered}
{\left[\frac{d^{2}}{d x^{* 2}}+\kappa^{2}-V\left(x^{*}\right)\right] H_{\omega}=0,} \\
\kappa=\frac{\omega}{c_{s 0}} \\
V\left(x^{*}\right)=\frac{1}{g^{2}}\left[\frac{g}{2} \frac{d^{2} g}{d x^{* 2}}-\frac{1}{4}\left(\frac{d g}{d x^{*}}\right)^{2}\right] .
\end{gathered}
$$

Here $c_{s 0}$ is the stagnation sound speed, and $x^{*}$ is an acoustic analogue of the tortoise coordinate which satisfies $x^{*}(x=+\infty)=+\infty, x^{*}(x=0)=-\infty$, namely,

$$
x^{*}=c_{s 0} \int \frac{d x}{c_{s}\left(1-M(x)^{2}\right)},
$$

where $M(x)$ is the Mach number [5], which, by the definition, is the current flow speed divided by the sound speed. In our notations $M=v / c_{s}$. The function $H_{\omega}$ represents small perturbations of gas flow,

$$
\begin{gathered}
H_{\omega}(x)=g^{1 / 2} \int d t e^{i \omega[t-f(x)]} \phi(t, x), \\
g=\frac{\sigma}{c_{s}} \\
f(x)=\int \frac{|v| d x}{c_{s}^{2}-v^{2}},
\end{gathered}
$$

Here, according to [4], the small perturbations are defined as follows

$$
\begin{aligned}
\rho & =\bar{\rho}+\delta \rho, & & \bar{\rho} \gg|\delta \rho|, \\
\Phi & =\bar{\Phi}+\phi, & & \left|\partial_{x} \bar{\Phi}\right| \gg\left|\partial_{x} \phi\right|,
\end{aligned}
$$

Our starting point is the calculation of the configuration of de Laval nozzle, i. e. its cross section as a function of the transversal nozzle coordinate. Since we know the effective potential, we can calculate in some way the function $g(x)$. By definition Eq. (15) of [4]

$$
g=\frac{\sigma}{c_{s}}=\frac{\rho A}{\sqrt{\gamma p / \rho}} .
$$

Taking (5) into account we find

$$
g \propto \frac{\rho A}{\rho^{(\gamma-1) / 2}} .
$$

We can choose dimensionless quantities for $\rho(x)$ and $A(x)$ by measuring them in units of $\rho_{0}$ and $A^{*}$ respectively [5]. Then equation (5.3) of [5] reads

$$
A^{-1} \propto\left(1-\rho^{(\gamma-1)}\right)^{1 / 2} \rho .
$$

Since (8) is invariant with respect to re-scaling of $g$, we can fix the coefficients in (17) and (18) arbitrarily:

$$
g=\frac{\rho A}{2 \rho^{(\gamma-1) / 2}}, \quad A^{-1}=\left(1-\rho^{(\gamma-1)}\right)^{1 / 2} \rho .
$$

We find

$$
g=\frac{\rho^{(1-\gamma) / 2}}{2\left(1-\rho^{(\gamma-1)}\right)^{1 / 2}}=\frac{\rho^{(1-\gamma)}}{2\left(\rho^{(1-\gamma)}-1\right)^{1 / 2}}
$$

Hence it follows that

$$
\rho^{1-\gamma}=2 g^{2}\left(1 \pm \sqrt{1-g^{-2}}\right) .
$$

The sign should be chosen in order that $\rho$ be a monotonous function with respect to the transverse coordinate. As we will show later, the function $g$ for the Schwarzschild black hole can be chosen also monotonous 
in the $R$ region, finite at the horizon and infinite at the spatial infinity. Therefore, we choose the minus sign,

$$
\rho^{1-\gamma}=2 g^{2}\left(1-\sqrt{1-g^{-2}}\right) .
$$

Note that $g$ must always be larger than unity in our consideration.

In our notations, the Mach number is connected with $\rho$ as

$$
\rho^{1-\gamma}=1+\frac{\gamma-1}{2} M^{2}
$$

We find

$$
\begin{gathered}
M^{2}=\frac{2}{\gamma-1}\left(\rho^{1-\gamma}-1\right)= \\
\frac{2}{\gamma-1}\left(2 g^{2}\left(1-\sqrt{1-g^{-2}}\right)-1\right) .
\end{gathered}
$$

Since $M=1$ at the event horizon, $g$ must be finite there, and

$$
\left.g\right|_{\text {e.h. }}=\frac{\gamma+1}{2 \sqrt{2} \sqrt{\gamma-1}}=\frac{3}{\sqrt{5}}>1
$$

This requirement fixes both constants of integration.

Substituting (23) in (19) we find the cross-section area as a function of $g$ :

$$
A=\frac{\sqrt{2}\left(2 g^{2}\left(1-\sqrt{1-g^{-2}}\right)\right)^{1 /(\gamma-1)}}{\sqrt{1-\sqrt{1-g^{-2}}}} .
$$

\section{THE DE LAVAL NOZZLE FOR THE SCHWARZSCHILD BLACK HOLE}

Our starting point is the calculation of the configuration of the de Laval nozzle, i. e. its cross section as a function of the transversal nozzle coordinate. Since we know the effective potential, we can calculate in some way the function $\mathrm{g}(\mathrm{x})$.

After separation of the angular and time variables, scalar field perturbations in the Schwarzschild background, can be reduced to the wave-like equation

$$
\left(\frac{d^{2}}{d r_{*}^{2}}+\omega^{2}-V\left(r^{*}\right)\right) \Psi\left(r^{*}\right)=0
$$

where putting the event horizon to be unity we find,

$$
\begin{gathered}
f(r)=1-\frac{1}{r}, \quad d r^{*}=\frac{d r}{f(r)} \\
V(r)=f(r)\left(\frac{\ell(\ell+1)}{r^{2}}+\frac{1-s^{2}}{r^{3}}\right)
\end{gathered}
$$

To find $g$ that produces the same potential we identify the "tortoise" coordinates of the black hole solution and of the laval nozzle

$$
\begin{gathered}
d r^{*}=d x^{*}=\frac{c_{s 0} d x}{c_{s}\left(1-M^{2}\right)}=\frac{\rho^{(1-\gamma) / 2} d x}{1-M^{2}}= \\
\frac{\sqrt{2 g^{2}\left(1-\sqrt{1-g^{-2}}\right)} d x}{1-\frac{2}{\gamma-1}\left(2 g^{2}\left(1-\sqrt{1-g^{-2}}\right)-1\right)} .
\end{gathered}
$$

Here $x$ is the real coordinate along de Laval nozzle. Then we can find the equation for $g(r)$,

$$
\frac{f(r) f^{\prime}(r) g^{\prime}(r)+f(r)^{2} g^{\prime \prime}(r)}{2 g(r)}-\frac{f(r)^{2} g^{\prime}(r)^{2}}{4 g(r)^{2}}=V(r) .
$$

This implies that the from of de Laval nozzle is parameterized by the parameter $r$ and $x^{*}(r)=r^{*}(r)$. Note that as we chose the radius of the event horizon to be unity, the nozzle coordinate $x$ is measured in the units of the radius of the event horizon.

The general solution of the equation (30) contains two arbitrary constants. They can be fixed in a unique way by the condition (25). Namely, the requirement that the solution must be finite at $r=1$ fixes one of the constant. Then the other constant re-scales the solution of (30), and must be fixed by its value at $r=1$. Finally, the solution of (30) for arbitrary $\ell$ and $s$, that satisfies (25) is given by the following formula:

$$
\begin{gathered}
g(r)= \\
\frac{\gamma+1}{2 \sqrt{2} \sqrt{\gamma-1}} \sum_{n=s}^{\ell}\left(\frac{(-1)^{n+s}(\ell+n) !}{(n+s) !(n-s) !(\ell-n) !} r^{n+1}\right)^{2}= \\
=\frac{\gamma+1}{2 \sqrt{2} \sqrt{\gamma-1}} r^{2 s+2} \times \\
\times\left(\frac{\Gamma(1+\ell+s)_{2} F_{1}(s-\ell, s+\ell+1,1+2 s, r)}{\Gamma(1+\ell-s) \Gamma(1+2 s)}\right)^{2} .
\end{gathered}
$$

One can easily check that the above solution indeed satisfies the equation (25), for any fixed $\ell$ and $s$.

From (29) we find the dependance of the transversal nozzle coordinate $x$ on the parameter $r$ :

$$
x=\int_{1}^{r} \frac{\left(\gamma+1-4 g(r)^{2}\left(1-\sqrt{1-g(r)^{-2}}\right)\right) d r}{f(r)(\gamma-1) \sqrt{2 g(r)^{2}\left(1-\sqrt{1-g(r)^{-2}}\right)}} .
$$

The integration constant is chosen in order to $x$ be zero at the sonic point.

Now we are in position to find the required form of de Laval nozzle. i.e. to find its cross-section $A(x)$. We just need to replace $g(r)$ given in (31) in (26) and go over to the transverse nozzle coordinate $x$. The function 
Figure 1: The form of de Laval nozzle and the effective potential for $s=\ell=0$.

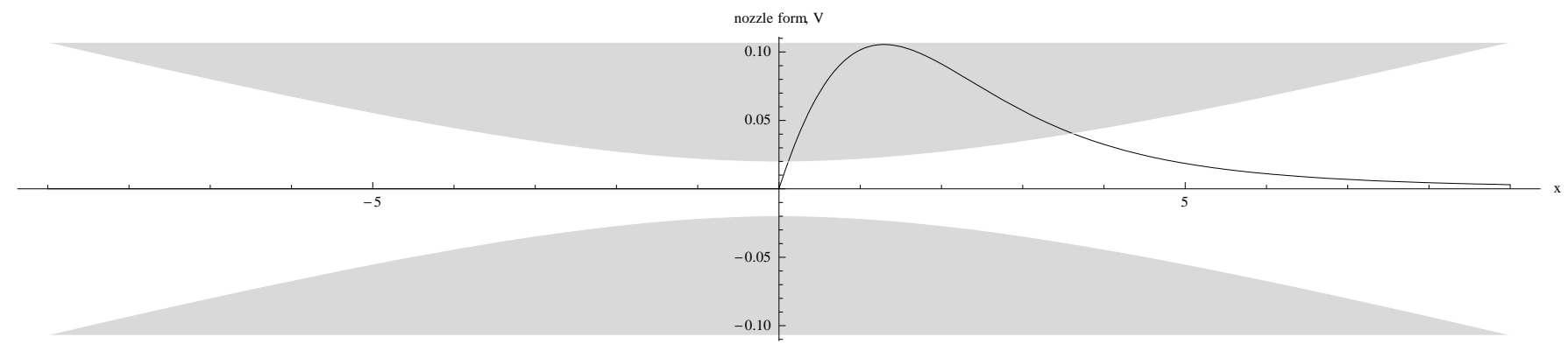

Figure 2: The form of de Laval nozzle and the effective potential for $s=\ell=1$.

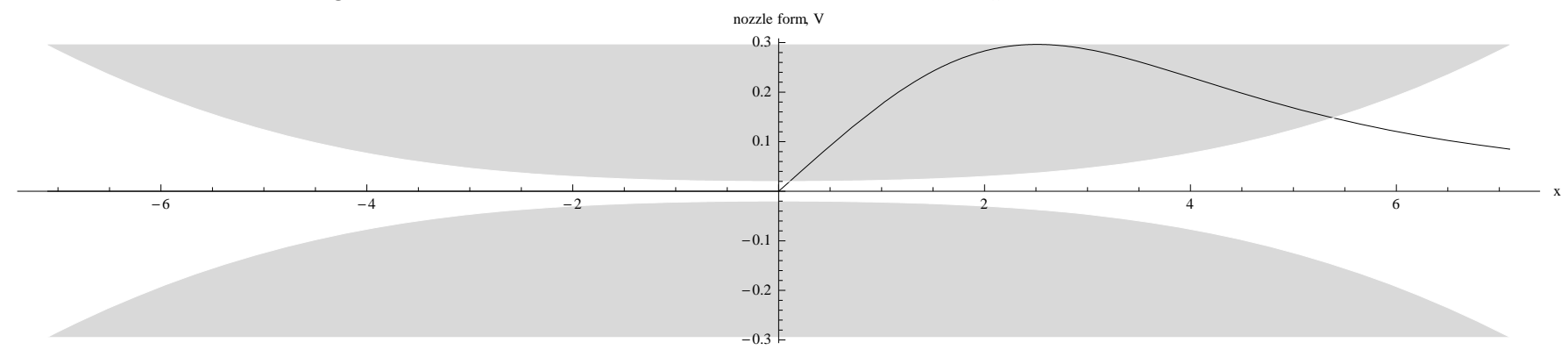

$A(x)$ is shown in Figs. 1 - 4. Note that the canonical de Laval nozzle is diverging at the end of the flow trajectory, so that $A_{x=\infty}=\infty$ (see pages 53 and 124 in [5]). Indeed, our formula (31) implies divergence at least as $\sim r^{2}$. The diverging of the nozzle nevertheless does not give any going beyond the one-dimensional representation of the motion, because the function $\sqrt{A(x)}$ is measured in units of black hole mass, i.e. one can "pull" the nozzle along the transverse coordinate $x$ in order to make the area of the nozzle change as slowly as one wishes. Such a "pulling" simply means that we are getting the correspondence with a black hole of larger mass. Since the quasinormal modes are inversely proportional to the mass of the black hole, this means just some determined multiplication by a coefficient when coming from the frequencies observed in experiment to the QNMs of a black hole.

As can be seen from [9], the values of the quasinormal modes are determined by the behavior of the effective potential in some region near black hole. The form of the effective potential (and thereby of de Laval nozzle) far from black hole is less significant for the QNMs problem. Therefore we expect that such experimental phenomena as surface friction and reflection of waves from boundaries will not have considerable influence on the observed picture.

Now we discuss the isospectrality and the effective potential for the polar and axial gravitational perturbations. We consider the effective potential for the gravitational perturbation of the polar type,

$$
V(r)=f(r) \frac{9(1+\lambda r)+\lambda^{3} r^{3}+\lambda^{2} r^{2}(3+2 r)}{r^{3}(3+\lambda)^{2}},
$$

where $\lambda=(\ell+2)(\ell-1)$.

For the above polar type gravitational perturbations we can also obtain the exact solution for the function $g(r)$, i.e. the form of de Laval nozzle. The function $g(r)$ is given in the following table for $\ell=2,3,4$, and 5 and in the formula (34):

$$
g(r, \ell)=\frac{\gamma+1}{2 \sqrt{2} \sqrt{\gamma-1}} \frac{r^{2} p(r, \ell)^{2}}{(3+(\ell+2)(\ell-1) r)^{2}}
$$

$$
\begin{array}{|l|l|}
\hline \ell & p(r, \ell) \\
\hline 2 & 3-6 r^{2}-4 r^{3} \\
3 & 3-30 r^{2}-20 r^{3}+60 r^{4} \\
4 & 3-90 r^{2}-60 r^{3}+630 r^{4}-504 r^{5} \\
5 & 3-210 r^{2}-140 r^{3}+3570 r^{4}-6552 r^{5}+3360 r^{6} \\
\hline
\end{array}
$$

Apparently there exists a solution for general $\ell$, yet probably quite cumbersome. Analysis of the Figs. 3-4 shows that the form of the nozzles for modeling polar and axial gravitational perturbations are almost the same. The difference cannot be seen explicitly, although not vanishing. As the effective potentials for axial and polar types also differ only slightly, this may mean that the forms of the nozzles also differ only slightly.

\section{DISCUSSION}

The suggested solution of the inverse problem for the correspondence of the form of de Laval nozzle to the general form of perturbations of the Schwarzschild black holes (i.e. for perturbations with $\operatorname{spin} s$ and multipole $\ell$ ) 
Figure 3: The form of de Laval nozzle and the effective potential for $s=\ell=2$.

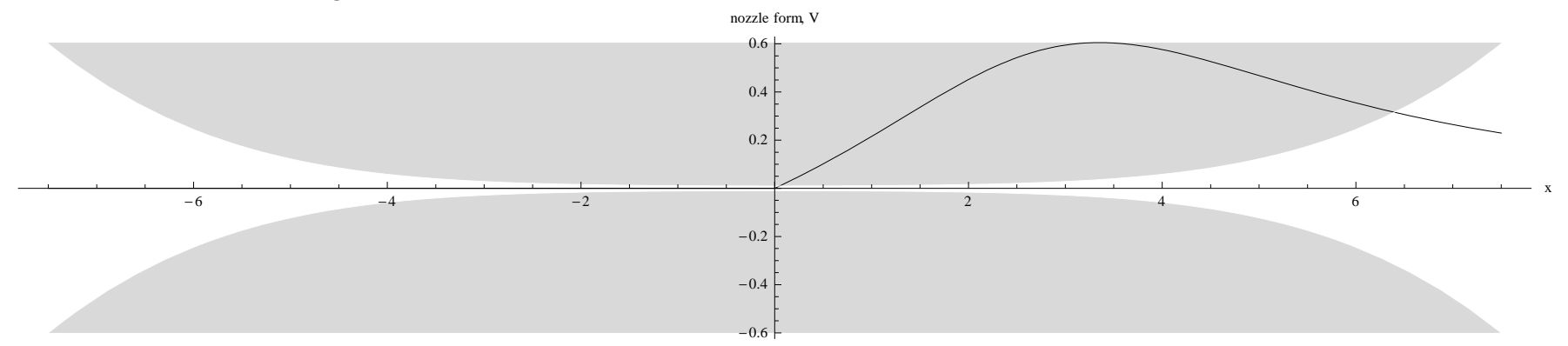

Figure 4: The form of de Laval nozzle and the effective potential for the polar gravitational perturbation $\ell=2$.

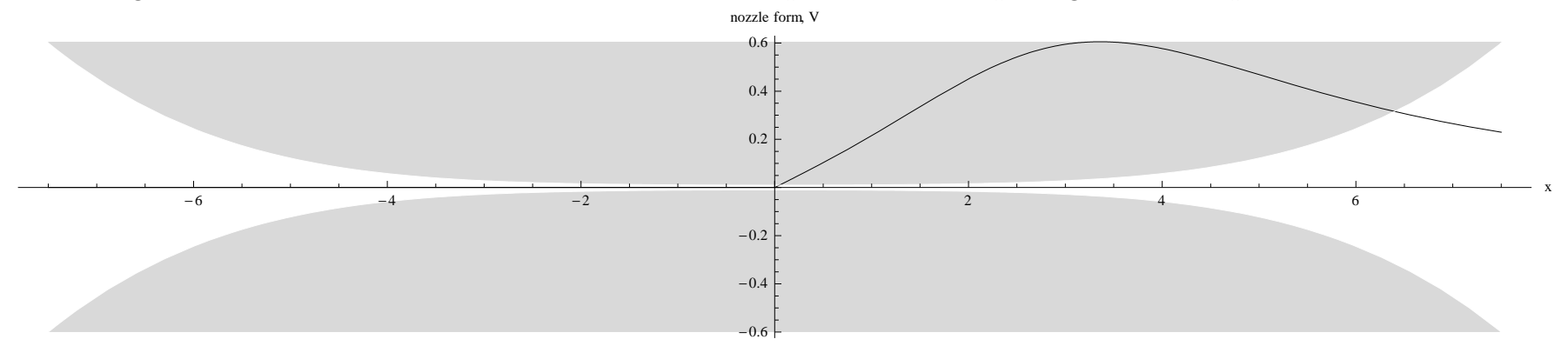

can be generalized in many ways. First of all, it would be very interesting to consider the massive vector [11] and scalar [10] field perturbations, because of quite unusual behavior of massive perturbations. Thus the so-called quasi-resonances, infinitely long lived modes, for massive scalar field [12] could be observed in a de Laval nozzle of some form as almost non-damping sound waves. These waves would certainly be reflected from the boundary of the nozzle and, thereby, would break the quasinormal mode boundary conditions. Even though in a real experiment one cannot obtain perfect QNM boundary condition (QNM b.c.), a considerable deviation from QNM b.c. should be observed when modeling the quasi-resonances.

Another possible generalization is to consider more general black hole backgrounds: ReissnerNordström, Schwarzschild-de Sitter, or higher dimensional Schwarzschild black holes [13] with charge, $\Lambda$-term and Gauss-Bonnet-term [15], including the brane-world black holes [14]. The Gauss-Bonnet black hole ringing [15] would be especially interesting to model in a nozzle because there exists the instability in some region of values of the black hole parameters 15]. In the approach considered in this paper we are limited only by a spherical symmetry, i.e. by $\omega$ independence of the effective potential. In addition, one could consider the flow of gas with a time dependent initial speed at the compressor, which probably could model the perturbations of the
Vaidya evaporating black holes [16], [17]. We cannot be sure, that in all these cases the differential equation for the form of de Laval nozzle will be exactly integrable, yet one can always find some numerical solution. We believe that further research will solve these interesting problems.

It should be recalled also that the precise acoustic analogy is only established for a scalar field. To be able to reproduce the potential $V(r)$ for fields of different spins certainly does not mean that one can reproduce all the characteristics of those equations in an acoustic model.

Finally, let us note that the obtained acoustic analogue for the perturbations of the Schwarzschild black holes is not limited by quasinormal mode problems only, but allow general investigation of propagation of classical and quantum fields, including such processes as scattering and tunnelling of waves and particles.

\section{Acknowledgments}

This work was supported by Fundação de Amparo à Pesquisa do Estado de São Paulo (FAPESP) and Conselho Nacional de Desenvolvimento Científico e Tecnológico $(C N P q)$, Brazil.
[1] K. D. Kokkotas and B. G. Schmidt, Living Rev. Relativity 2, 2 (1999).
[2] J. M. S. Zhu, B. Wang and E. Abdalla, Phys. Rev. D 63, 124004 (2001) arXiv:hep-th/0101133; H. 
c. Kao, arXiv:0704.0284 [hep-th]; Q. Y. Pan and J. L. Jing, Mod. Phys. Lett. A 21, 2671 (2006); C. Ma, Y. Gui, W. Wang and F. Wang, arXiv:gr-qc/0611146 Y. Zhang and Y. X. Gui, Class. Quant. Grav. 23, 6141 (2006) arXiv:gr-qc/0612010; S. Musiri and G. Siopsis, arXiv:hep-th/0610170 R. A. Konoplya, J. Phys. Stud. 8, 93 (2004); D. Birmingham, Phys. Lett. B 569, 199 (2003) arXiv:hep-th/0306004; R. A. Konoplya, Phys. Rev. D 68, 024018 (2003) arXiv:gr-qc/0303052; L. Motl, Adv. Theor. Math. Phys. 6, 1135 (2003) arXiv:gr-qc/0212096.

[3] W. G. Unruh, Phys. Rev. D 51, 2827 (1995); W. G. Unruh, Phys. Rev. Lett. 46, 1351 (1981)

[4] S. Okuzumi and M. a. Sakagami, arXiv:gr-qc/0703070

[5] H. W. Liepmann and A. Roshko, Elements of Gasdynamics (John Wiley \& Sons, 1957), Chap. 5.

[6] V. Cardoso, J. P. S. Lemos and S. Yoshida, Phys. Rev. D 70, 124032 (2004) arXiv:gr-qc/0410107); E. Berti, V. Cardoso and J. P. S. Lemos, Phys. Rev. D 70, 124006 (2004) arXiv:gr-qc/0408099; C. Barcelo, A. Cano, L. J. Garay and G. Jannes, Phys. Rev. D 75, 084024 (2007) arXiv:gr-qc/0701173; J. Saavedra, Mod. Phys. Lett. A 21, 1601 (2006) arXiv:gr-qc/0508040; C. Cherubini, F. Federici, S. Succi and M. P. Tosi, Phys. Rev. D 72, 084016 (2005) arXiv:gr-qc/0504048; H. Nakano, Y. Kurita, K. Ogawa and C. M. Yoo, Phys. Rev. D 71, 084006 (2005) arXiv:gr-qc/0411041;

S. Lepe and J. Saavedra, Phys. Lett. B 617, 174 (2005) arXiv:gr-qc/0410074.

[7] X. H. Ge and S. W. Kim, arXiv:0705.1396 [hep-th].

[8] X. H. Ge and S. W. Kim, arXiv:0705.1404 [hep-th].

[9] R. A. Konoplya and A. Zhidenko, Phys. Lett. B 648, 236 (2007) arXiv:hep-th/0611226; R. A. Konoplya and A. Zhidenko, Phys. Lett. B 644, 186 (2007) arXiv:gr-qc/0605082.

[10] L. E. Simone and C. M. Will, Class. Quant. Grav. 9, 963 (1992); R. A. Konoplya, Phys. Lett. B 550,
117 (2002) arXiv:gr-qc/0210105; T. Jana and P. Roy, arXiv:0705.3304 |gr-qc|.

[11] R. A. Konoplya and C. Molina, Phys. Rev. D 75, 084004 (2007) arXiv:gr-qc/0602047; R. A. Konoplya, Phys. Rev. D 73, 024009 (2006) arXiv:gr-qc/0509026.

[12] A. Ohashi and M. a. Sakagami, Class. Quant. Grav. 21, 3973 (2004).

[13] V. Cardoso, O. J. C. Dias and J. P. S. Lemos, Phys. Rev. D 67, 064026 (2003); R. A. Konoplya and A. Zhidenko, arXiv:hep-th/0703231; R. A. Konoplya, Phys. Rev. D 68, 124017 (2003) arXiv:hep-th/0309030; A. LopezOrtega, Gen. Rel. Grav. 38, 1565 (2006); A. Saa, arXiv:gr-qc/0701153; H. T. Cho, A. S. Cornell, J. Doukas and W. Naylor, arXiv:hep-th/0701193.

[14] P. Kanti, R. A. Konoplya and A. Zhidenko, Phys. Rev. D 74, 064008 (2006) arXiv:gr-qc/0607048; J. y. Shen, B. Wang and R. K. Su, Phys. Rev. D 74, 044036 (2006); P. Kanti and R. A. Konoplya, Phys. Rev. D 73, 044002 (2006) arXiv:hep-th/0512257]; E. Abdalla, B. CuadrosMelgar, A. B. Pavan and C. Molina, Nucl. Phys. B 752, 40 (2006). arXiv:gr-qc/0604033.

[15] G. Dotti and R. J. Gleiser, Phys. Rev. D 72, 044018 (2005); R. Konoplya, Phys. Rev. D 71, 024038 (2005) arXiv:hep-th/0410057; E. Abdalla, R. A. Konoplya and C. Molina, Phys. Rev. D 72, 084006 (2005) arXiv:hep-th/0507100; R. J. Gleiser and G. Dotti, arXiv:gr-qc/0510069; S. K. Chakrabarti, Gen. Rel. Grav. 39, 567 (2007); F. Moura and R. Schiappa, Class. Quant. Grav. 24, 361 (2007); R. G. Daghigh, G. Kunstatter and J. Ziprick, Class. Quant. Grav. 24, 1981 (2007); K. Konya, Phys. Rev. D 75, 104003 (2007) arXiv:hep-th/0701257.

[16] E. Abdalla, C. B. M. Chirenti and A. Saa, arXiv:gr-qc/0703071.

[17] E. Abdalla, C. B. M. Chirenti and A. Saa, Phys. Rev. D 74, 084029 (2006) arXiv:gr-qc/0609036. 